\title{
Risco de quedas pela fragilidade em idosos comunitários praticantes de atividade física
}

\author{
Risk of falls by frailty in community-dwelling elderly practitioners of \\ physical activity
}

Gabriel Pereira da Costa ${ }^{1}$, Adriana Sousa Rego ${ }^{1}$, Flor da Maria Araújo Mendonça Silva $^{2}$, Laíse Neves Carvalho ${ }^{1}$ José Newton Lacet Vieira ${ }^{3}$

\begin{abstract}
Resumo: Objetivo: Identificar características de risco de quedas pela fragilidade em idosos comunitários praticantes de atividade física. Materiais e métodos: Tratou-se de um estudo descritivo de corte transversal, realizado em maio de 2016 com idosos da Associação Comunitária da Vila Cruzado. Os componentes de fragilidade analisados foram força muscular máxima de preensão manual, com uso do Dinamômetro de Preensão Manual Palmar, avaliação do estado mental, através no Mini Exame do Estado Mental e do medo de cair, por meio da Falls Eficacy Scale-International-Brasil Total. Este estudo foi aprovado pelo Comitê de Ética em Pesquisa em Humanos da Universidade CEUMA, com protocolo no 1.516.257/2016. Para análise dos dados foi usado o programa estatístico SPSS 18.0. Resultados: Foram inseridos no estudo 19 idosos. A mediana da força muscular manual foi de $4 \mathrm{Kgf}$, sendo 0 mínimo $0 \mathrm{Kgf}$ e o máximo $19 \mathrm{Kgf}$. Em relação ao estado mental, 94,7\% (18) apresentaram déficit cognitivo leve e apenas $5,3 \%$ (1) déficit moderado. Referente a queda, 36,8\% (7) relataram medo acentuado de cair, $57,9 \%$ (11) moderado e $5,3 \%$ (1) não relatou medo. Conclusão: Apesar das limitações do estudo transversal, sugere-se que a atividade física diminui o risco de quedas pela fragilidade em idosos comunitários.
\end{abstract}

Palavras-chave: Força muscular. Cognição. Acidentes por quedas. Idoso.

Abstract: Objective: To identify risk characteristics of falls by the weakness in physically active elderly community. Materials and methods: This was a descriptive cross-sectional study, conducted in May 2016 with the elderly of the Community Association of the Village Cross. The fragility analyzed components were maximal muscle strength of handgrip, with use of Dynamometer Trapping Manual Palmar, assessment of mental state by the Mini-Mental State Examination and of the fear of falling through the Falls Eficacy Scale- International- Brazil Total. This study was approved by the Ethics Committee on Human Research of the University CEUMA with Protocol 1516257 / 2016. For data analysis was used SPSS 18.0. Results: They were enrolled in the study 19 elderly. The median manual muscle strength was $4 \mathrm{kgf}$, the minimum and maximum $0 \mathrm{kgf} 19 \mathrm{kgf}$. Regarding mental status, $94.7 \%(18)$ had mild cognitive impairment and only $5.3 \%$ (1) moderate deficit . Regarding the fall, $36.8 \%$ (7) reported sharp fear of falling, $57.9 \%$ (11) moderate and $5.3 \%$ (1) reported no fear. Conclusion: It was concluded that despite the limitations of cross-sectional study, it is suggested that physical activity decreases the risk of falls by the weakness in community-dwelling.

Keywords: Muscle strength. Cognition. Accidental falls. Aged.

\footnotetext{
${ }^{1}$ Departamento de Fisioterapia. Universidade Ceuma

2 Departamento de Psicologia. Universidade Ceuma

${ }^{3}$ Departamento de Fisioterapia. Universidade Ceuma.

Endereço para correspondência: Avenida Nova lorque, Q-5 E, C-03, Central Parque, Araçagy, São José de Ribamar-MA. CEP: 65.110-000. Fone: (98) 981109633.
} 


\section{Introdução}

O aumento acentuado do número de idosos nas últimas décadas e o fato de grande parte deles permanecer em atividade e com autonomia fizeram com que o interesse pelo estudo do envelhecimento fosse se dando progressivamente. ${ }^{1}$

Segundo Cruz et al. ${ }^{2}$, o envelhecimento biológico compromete de forma distinta 0 funcionamento de todos os sistemas do organismo, sendo o Sistema Nervoso Central um dos mais afetados pelo processo de senescência, com o desenvolvimento de alterações no sistema de neurotransmissores e hipotrofia cerebral. Essas modificações acontecem preferencialmente nas regiões responsáveis pelas funções cognitivas, como os lobos frontal e temporal, incluindo o sistema límbico. Dessa forma, é comum observar desde mínimos até significativos prejuízos da função cognitiva entre os indivíduos idosos.

O aumento da expectativa de vida desencadeou mudanças nos perfis demográficos e epidemiológicos da população brasileira, o que resultou no crescimento no número de idosos. Esta condição tem relação com a diminuição das doenças transmissíveis, contrapondo-se a elevação das crônicas e nãotransmissíveis. ${ }^{3}$

Estudos de prevenção de quedas e avaliação de riscos têm sido um dos principais focos de saúde pública e cuidados de enfermagem. Para evitar quedas na vida diária, medidas tais como: 0 exercício, o treinamento muscular, andar com uma bengala, ou a renovação de seu ambiente para remover impedimentos são importantes. ${ }^{4}$

Uma das maiores
preocupações com relação à saúde dos idosos é a elevada taxa de quedas, que são muitas vezes causa de morbimortalidade. Quase metade das internações hospitalares de idosos no Brasil é motivada por quedas. A capacidade de manter a estabilidade postural pode ser afetada pela associação de tarefas cognitivas, principalmente se estas forem complexas. Quando há maior demanda cognitiva, como ao manter o equilíbrio e executar uma tarefa simultaneamente, o desempenho é prejudicado, especialmente em pessoas com história de quedas. ${ }^{5}$

Os primeiros estudos relacionavam fragilidade diretamente ao declínio funcional, provavelmente devido à falta de um foco clínico em questão. Como fragilidade não é uma queixa clínica e sua presença é freqüentemente sutil ou assintomática, embora se torne evidente ao longo do tempo, as queixas sempre estavam relacionadas com incapacidade funcional. ${ }^{6}$

Segundo Martin et al. $^{7}$, Recentemente, um emergente foco foi colocado em estudar os efeitos da deficiência em domínios cognitivos específicos, como a função executiva, atenção e memória. A lógica é que declínio da função cognitiva ocorre de modo não uniforme em todos os domínios e níveis e os estudos de habilidades cognitivas específicas podem elucidar melhor os caminhos que levam a quedas. 
$\begin{array}{ccc}\text { Estudos } & \text { mostraram a } \\ \text { importância da estratégia de }\end{array}$ controle postural biomecânico, refletida nos ajustes posturais das articulações dos membros inferiores, na compreensão da relação entre marcha e quedas. Observaram que os elevados momentos de força do joelho e quadril produzidos durante 0 desequilíbrio na marcha indicam a influência dessas articulações na recuperação do equilíbrio, e que o tornozelo atua apenas passivamente na tentativa de evitar a queda. ${ }^{8}$

Segundo Piovesan et al. ${ }^{9}$, a fisioterapia surge como uma área de conhecimento que desperta seu olhar para a saúde do idoso, buscando manter e melhorar a funcionalidade, com vistas à autonomia do sujeito e a qualidade de vida. A fisioterapia desempenha importante papel na prevenção das quedas, pois proporciona melhora do quadro motor e do equilíbrio, além de fornecer orientações aos pacientes e seus cuidadores, eliminando ou minimizando os fatores de risco.

Considerando que o estudo sobre a população idosa é importante por tratar-se de uma população crescente, e que a fisioterapia é uma ciência que atua na promoção de saúde e na proteção específica; este estudo tem como objetivo identificar características de risco de quedas pela fragilidade em idosos comunitários praticantes de atividade física.

\section{Materiais e métodos}

Tratou-se de um estudo descritivo de corte transversal. ${ }^{10} \mathrm{~A}$ população foi composta por 19 idosos, por conveniência, com idade igual ou superior a 60 anos, dos sexos masculino e feminino, no mês de maio de 2016, na Associação Comunitária da Vila Cruzado (ACOVIC).

Para coleta de dados foi feita inicialmente uma reunião com os idosos comunitários.

Em seguida, após a seleção das idosas por critérios de inclusão e de exclusão, foi utilizado o Mini Exame do Estado Mental (MEEM), desenvolvido por Folstein et al. ${ }^{11}$. E um dos testes mais empregados $e$ mais estudados em todo o mundo. Usado isoladamente ou incorporado a instrumentos mais amplos, permite a avaliação da função cognitiva e rastreamento de quadros demenciais.

Foi utilizada também a entrevista por meio da Falls Eficacy Scale- International-Brasil Total (FES-I-Brasil Total), versão adaptada e validada para o Brasil, por Camargos et al. ${ }^{12}$, para avaliação do medo de cair.

E verificada a avaliação da força muscular máxima de preensão manual, com uso do Dinamômetro de Preensão Manual Palmar, da marca Sanny com resolução em Kgf, portátil, em aço Inox, com capacidade de 99 kgf, sendo utilizado pela mão dominante, como um indicador da força total do corpo, empregado em testes de aptidão física, e fácil de ser utilizado. ${ }^{13}$ Durante a avaliação da força de preensão manual, cada idosa foi posicionada sentada em uma cadeira, utilizando 0 membro 
superior dominante apoiado, com o antebraço em posição neutra, e cotovelo flexionado a $90^{\circ}$ ADM. Ao comando do avaliador, cada idosa realizou a contração muscular isométrica resistida, dos flexores dos dedos, com duração de 5 segundos. Foram realizadas três repetições com intervalo de um minuto. Foi considerada para a avaliação das idosas, a força muscular máxima de preensão manual em Kgf, das três repetições.

Este estudo foi baseado na resolução 466/12 do Conselho Nacional de Saúde que regulamenta as pesquisas envolvendo seres humanos, ${ }^{14}$ respeitando-se a Convenção de Helsinki em 2008; ${ }^{15}$ tendo sido aprovado pelo Comitê de Ética em Pesquisa em Humanos da Universidade CEUMA, com protocolo $\mathrm{n}^{\circ}$ 1.516.257/2016.

Os dados referentes à coleta de dados foram tabulados em uma planilha do programa Microsoft Office Excel 2010®, e transferidos para análise com 0 programa estatístico Statistical Package for the Social Sciences (SPSS), versão 18.0 .

$\mathrm{Na}$ estatística descritiva, as variáveis quantitativas com distribuição assimétrica são apresentadas em mediana, mínimo e máximo, enquanto que as variáveis qualitativas foram apresentadas por meio de frequências absolutas, e percentuais.

\section{Resultados}

Foram inseridos no estudo 19 idosos. A mediana da força muscular manual foi de $4 \mathrm{Kgf}$, sendo o mínimo $0 \mathrm{Kgf}$ e o máximo $19 \mathrm{Kgf}$ (figura 1).

Em relação ao estado mental, $95 \%$ (18) apresentaram déficit cognitivo leve e apenas $5 \%$ (1) déficit moderado (figura 2).

Referente a queda, 37\% (7) relataram medo acentuado de cair, $58 \%$ (11) moderado e 5\% (1) não relatou medo (figura 3 ).

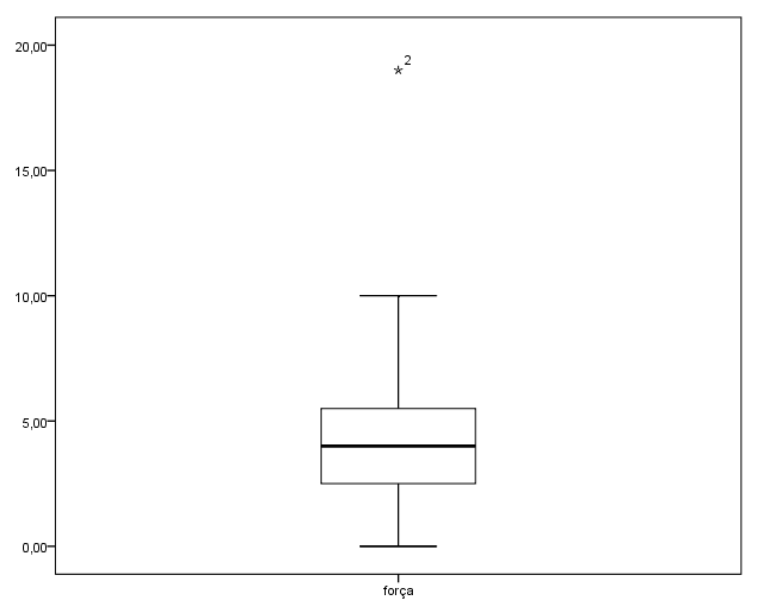

Figura 1 - Força muscular manual de idosos comunitários praticantes de atividade física. São Luís -MA, 2016 


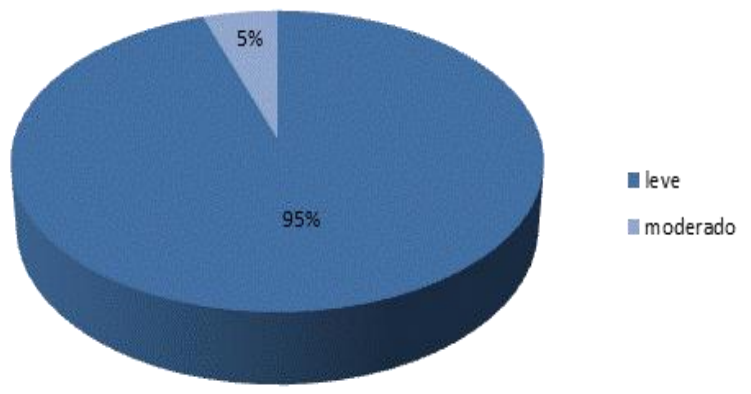

Figura 2 - Estado mental de idosos comunitários praticantes de atividade física. São Luís -MA, 2016

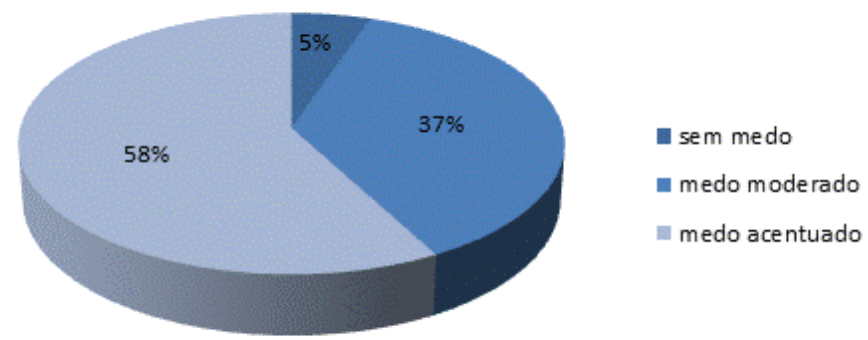

Figura 3 - Medo de queda de idosos comunitários praticantes de atividade física. São Luís -MA, 2016

\section{Discussão}

Estudo realizado por Hauser et al. ${ }^{16}$, identificou que as capacidades físicas como força, parecem ser fatores decisivos em relação à preocupação com a possibilidade de cair em idosos praticantes de atividade física. Corroborando com este estudo que mostrou ser importante a prática de atividade física para o ganho de força muscular, apresentado na figura 1.
Conforme Camargos et al. ${ }^{12} \mathrm{o}$ medo de cair é um problema de saúde comum e significativo que pode levar ao declínio funcional, mas é potencialmente modificável, estando de acordo com este estudo que apresentou que idosos praticantes de atividade física apresentam medo de cair, e por tal motivo buscam uma solução preventiva para este problema, identificado na figura 2 . 
Este estudo mostrou que os idosos apresentam algum déficit cognitivo, sendo importante a prática de uma atividade física que possa contribuir para evitar um maior risco de queda, verificado no gráfico 3. Estando de acordo com o estudo realizado por Valcarenghi et al. ${ }^{17}$, que percebeu que, dos 25 idosos aos quais foi possível aplicar o MEEM, 20 apresentaram 0 em pontuações entre 23 ou menos, representando indicação para deficit cognitivo; desses, dez residentes relataram a ocorrência de quedas.

\section{Conclusão}

Concluiu-se neste estudo que apesar das limitações do estudo transversal, sugere-se que a atividade física diminui o risco de quedas pela fragilidade em idosos comunitários.

\section{Referências}

1. Lojudice DC, Laprega MR, Rodrigues RAP, Rodrigues AL Jr. Quedas em idosos institucionalizados: ocorrências e fatores associados. Rev Bras Geriatr Gerontol. 2010;13(3):40312.

2. Cruz DT, Cruz FM, Ribeiro CLV, Leite IC. Associação entre capacidade cognitiva e ocorrência de quedas em idosos. Cad Saúde Colet. 2015;23(4):386-93.

3. Leite MT, Hildebrandt LM, Kirchner RM, Winck T, Silva LAA, Franco GP. Estado cognitivo e condições de saúde de idosos que participam de grupos de convivência. Rev Gaúcha Enferm. 2012;33(4):64-71.
4. Akahane $M$, Maeyashiki $A$, Yoshihara S, Tanaka Y, Imamura $T$. Relationship between difficulties in daily activities and falling: lococheck as a self-assessment of fall risk. Interact J Med Res. 2016;5(2):e20.

5. Custódio EB, Malaquias J Jr, Voos MC. Relação entre cognição (função executiva e percepção espacial) e equilíbrio de idosos de baixa escolaridade. Fisioter Pesq. 2010;17(1):46-51.

6. Santos EGS. Perfil de fragilidade em idosos comunitários de Belo Horizonte: um estudo transversal [dissertação]. Belo Horizonte: Universidade Federal de Minas Gerais; 2008.

7. Martin KL, Blizzard L, Srikanth VK, Wood A, Thomson R, Sanders LM, et al. Cognitive function modifies the effect of physiological function on the risk of multiple falls--a population-based study. J Gerontol A Biol Sci Med Sci. 2013 Sep;68(9):1091-7.

8. Jacquemin JSA, Santos P, Garcia PA, Dias RC, Dias JMD. Comparação da função muscular isocinética dos membros inferiores entre idosos caidores e não caidores. Fisioter Pesq. 2012;19(1):39-44.

9. Piovesan AC, Pivetta HMF, Peixoto JMB. Fatores que predispõem a quedas em idosos residentes na região oeste de Santa Maria, RS. Rev Bras Geriatr Gerontol. 2011;14(1):75-83.

10. Thomas JR, Nelson JK, Silverman SJ. Métodos de pesquisa em atividade física. 5 $5^{\mathrm{a}}$ ed. Porto Alegre: Artmed; 2007.

11. Folstein MF, Folstein SE, Mchugh PR. Mini Mental state a practical 
method for grading and cognitive state of patients for the clinician. $J$ Psychiatr Res. 1975;12(3):189-98.

12. Camargos FFO, Dias RC, Dias JMD, Freire MTF. Adaptação transcultural e avaliação das propriedades psicométricas da Falls Efficacy Scale-International: um instrumento para avaliar medo de cair em idosos. Rev Bras Fisioter. 2010;14(3):237-43.

13. Rebellato JR, Castro AP, Chan A. Quedas em idosas institucionalizados: características gerais, fatores determinantes e relações com a força de preensão manual. Acta Ortop Bras. 2007;15(3):151-4.

14. Conselho Nacional de Saúde. Resolução no 466, de 12 de dezembro de 2012. Homologa a Resolução CNS nำ 466, de 12 de dezembro de 2012, nos termos do decreto de Delegação de Competência de 12 de novembro de 1991. Diário da República Federativa do Brasil, Brasília (DF); 2013 jun 13; Seção 1:59.

15. World Medical Association. World Medical Association Declaration of Helsinki: ethical principles for medical research involving human subjects. 59 ${ }^{\text {th }}$ ed. Seoul: WMA General Assembly; 2008.

16. Hauser E, Sandreschi PF, Parizzotto D, Araújo CCR, Mazo GZ. Medo de cair e desempenho físico em idosos praticantes de atividade física. Rev Educ Fís/UEM. 2015;26(4):593-600.

17. Valcarenghi RV, Santos SSC, Barlem ELD, Pelzer MT, Gomes GC, Lange C. Alterações na funcionalidade/cognição depressão em idosos institucionalizados que sofreram quedas. Acta Paul Enferm. $2011 ; 24(6): 828-33$. 